\title{
Präferenzen, Nutzen und ihre Aggregation
}

\author{
Christoph Lumer
}

\section{Inhalt}

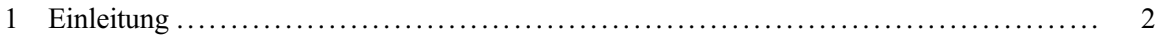

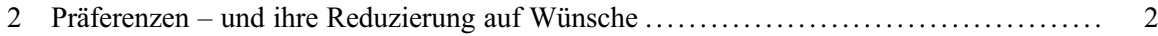

3 Wünsche - motivationale Funktion, Mechanik und ihr kognitiver Gehalt ............. 3

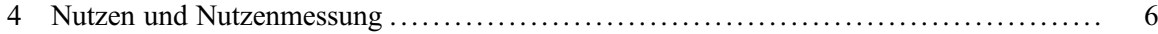

5 Intrinsischer Nutzen und intrinsische Wünschbarkeit .......................... 9

6 Nutzenaggregation als Definition des, Gemeinwohls' ${ }^{\text {' }}$ und des , moralischen Werts ${ }^{6}$....... 10

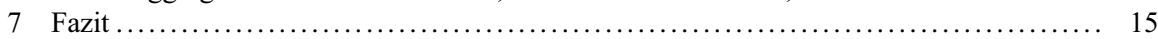

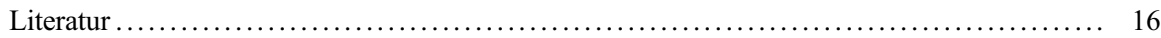

\section{Zusammenfassung}

,Wunsch“, ,Präferenz‘, ,Nutzen“, ,(nutzenaggregierende) moralische Wünschbarkeit' sind Begriffe, die in dieser Reihenfolge aufeinander aufbauen. Der Artikel folgt diesem definitorischen Aufbau und stellt diese Begriffe sowie die zugehörigen Begründungen vor. Der Zielpunkt ist die Darstellung von wohlfahrtsethischen Kriterien des Gemeinwohls, die die ,moralische Wünschbarkeit' als Aggregation, z. B. Addition, von individuellen Nutzen definieren: Utilitarismus, Nutzenegalitarismus, Leximin, Prioritarismus.

\section{Schlüsselwörter}

Präferenz · Nutzen · Moralische Wünschbarkeit · Utilitarismus · Prioritarismus

C. Lumer $(\triangle)$

Universität Siena, Siena, Italien

E-Mail: lumer@unisi.it 


\section{$1 \quad$ Einleitung}

Thema dieses Artikels sind schlussendlich verschiedene ethische, genauer: wohlfahrtsethische, Vorschläge für eine quantitative Definition von ,Gemeinwohl', nämlich diverse Theorien und Definitionen des ,moralischen Werts ${ }^{6}$ oder der ,moralischen Wünschbarkeit ${ }^{6}$ - wie die utilitaristische oder egalitaristische oder prioritaristische Definition und Theorie. Das Besondere an diesen Definitionen und Theorien ist, dass sie alle die moralische Wünschbarkeit als eine spezielle Form der Aggregation (also Zusammenstellung) aus den individuellen/prudentiellen Nutzen aller Betroffenen ansehen, im einfachsten Fall als Summe aller dieser individuellen Nutzen. Dadurch kann die so definierte ,moralische Wünschbarkeit" wirklich das Gemeinwohl darstellen, in dem eben das (individuelle) Wohl aller berücksichtigt wird. Die zusätzlichen Vorteile eines solchen quantitativen Begriffs des Gemeinwohls sind, dass damit Alternativen in der für die Entscheidung relevanten Hinsicht sehr einfach verglichen werden können und diverse Kombinationen mehrerer Maßnahmen bewertet werden oder die moralische Effizienz (möglichst viel moralische Wünschbarkeit pro Einheit an investierten Ressourcen) diverser Optionen bestimmt werden kann, um mit den vorhandenen Ressourcen möglichst viel Gemeinwohl schaffen zu können.

Die Definition der ,moralischen Wünschbarkeit‘ setzt also den Begriff des ,individuellen/prudentiellen Nutzens` voraus. Dieser wiederum lässt sich über den der ,(individuellen) Präferenz' (des entsprechenden Individuums) definieren. Die Darstellung hier wird der systematischen Reihenfolge dieser Begriffe vom Einfachen zum Komplexen folgen, also den Präferenzbegriff zuerst behandeln, dann den des individuellen Nutzens und schließlich Nutzenaggregationen und die moralische Wünschbarkeit thematisieren.

Terminologisch wird hier kein Unterschied zwischen ,sozialer/moralischer Wünschbarkeit' und ,sozialem/moralischem Wert' gemacht, ebensoweníg zwischen ,individueller/prudentieller Wünschbarkeit', ,individuellem/prudentiellem Nutzen' und ,individuellem/prudentiellem Wert‘. Es werden aber vorzugsweise die Formulierungen „moralische Wünschbarkeit“ und ,prudentieller Nutzen“ verwendet, so dass dann die Adjektive gelegentlich auch weggelassen werden können.

\section{Präferenzen - und ihre Reduzierung auf Wünsche}

Eine Präferenz i.e.S. ist eine subjektive Einstellung der Art, dass ein Subjekt $s$ einen Sachverhalt $p$ einem Sachverhalt $q$ vorzieht, $p$ also als besser ansieht. Der Präferenzbegriff ist komparativ: es werden zwei Dinge in einer bestimmten Dimension miteinander verglichen. Wie bei allen Beschreibungen propositionaler Einstellungen sind die Argumente für die verglichenen Sachverhalte $p$ und $q$ intensional.

Der komparative Präferenzbegriff hat bis zur Entstehung der axiomatisierten rationalen Entscheidungstheorie philosophisch keine Rolle gespielt; sondern es wurde stattdessen fast ausschließlich der vage quantifizierende Wunschbegriff

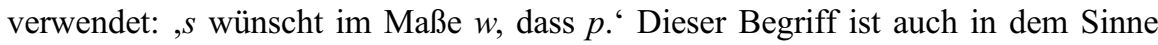


phänomenal ursprünglicher, dass uns unsere Wünsche unmittelbarer zugänglich sind - obwohl auch diese bei weitem nicht immer natürlich oder spontan sind, sondern oft epistemisch gebildet werden - und dass Präferenzen oft erst durch den Vergleich zweier Wunschstärken gebildet werden: Wenn wir $p$ im Maße $w$ wünschen und $q$ im Maße $v$, zudem $w$ als größer ansehen als $v$, dann präferieren wir anschließend $p$ vor $q$. Präferenzen sind also schon abgeleitet und können über die beiden Wünsche begründet werden (ausführliche Begründung: Schroeder 2020, Abschn. 3.3). Wegen der Reduzierbarkeit der Präferenzen auf Wünsche gelten viele der im Folgenden vorgenommenen Differenzierungen für beide - auch wenn, der Einfachheit halber, häufig nur von den Wünschen die Rede ist.

\section{Wünsche - motivationale Funktion, Mechanik und ihr kognitiver Gehalt}

Der Ausdruck „Wunsch“ (und seine Pendants in anderen westeuropäischen Sprachen) hat philosophiegeschichtlich und alltagssprachlich 1. eine phänomenale, emotionale und 2. eine funktional-erklärende Bedeutungslinie mit Bedeutungsclustern um die Begriffe 1. ,begehren', ,(er)sehnen', ,Mangelerfahrung ' bzw. 2. ,Handlungstendenz', ,Neigung', ,streben' (vgl. Fehige und Weiß 2004, Sp. 1078). In der deutschen Alltagssprache ist die phänomenale Bedeutung dominant (Grimm und Grimm 1991, Sp. 2020, 2029, 2042, 2048). In der Philosophie, in der Handlungs- und rationalen Entscheidungstheorie, ist seit etwa 1950 ,Wunsch", ,wünschen“ (englisch: „desire“, aber nicht „wish“) ein eher technischer Begriff, in dem die funktional-erklärende Bedeutung dominant ist (z. B. Davidson 1980a, S. 3-4, 1980b, S. 86-87 (spricht von „Pro-Einstellung“, wozu insbesondere ,desires“ und „wants" gehören); Brandt 1979, S. 24-26 (spricht von ,wants“ und setzt dies gleich mit ,positive valence“); Mele 1992, S. 72, 77, 177; Schueler 1995, S. 21-35; zusammenfassend: Fehige und Weiß 2004, Sp. 1080-1083). Gemäß dieser technischen Bedeutung implizieren von den mentalen Zuständen, die damit erfasst werden, die phänomenalen generell auch die erklärenden, aber nicht umgekehrt: Es gibt auch sich rein kognitiv anfühlende Wünsche im Sinne von „Strebungen“ ohne jede emotionale Komponente - man denke etwa an einen Aktienhändler, der eine Fülle von Investmententscheidungen im Sinne der Gewinnmaximierung fällt.

Wenn eine Handlungserklärung mittels Wünschen nicht behavioristisch sein soll, dann muss es außer der bloßen Handlungstendenz auch einen subjektiven, phänomenalen Zustand geben, der eigentlich dieser Wunsch ist und die Handlungstendenz verursacht. Und nach dem technischen Wunschbegriff kann dieser subjektive Zustand nicht in einer affektiven Komponente des Sehnens oder angenehmer Gefühle beim Denken an das Gewünschte bestehen - auch wenn er zuweilen von solchen Affekten begleitet wird. Vielmehr ist dieser subjektive Zustand glaubensgleich, kann als Glaube eines bestimmten Inhalts repräsentiert werden, nämlich als Werturteil: ,(Der Gegenstand) $p$ ist $u /$ so und so (sehr, ein bisschen ...) gut $/$ schlecht (für mich)'; das Argument $u$ gibt die Wünschbarkeit (psychologisch: „Valenz“) des Wertobjekts an. (,The natural expression of his desire is [...] evaluative in form; for example, ,It is desirable to improve the taste of the stew “" (Davidson 1980b, S. 86)). Dieses 
Werturteil hat aber - nur bei dieser Art von Inhalten und Bewertungskriterien - eine über den Glauben hinausgehende motivationale Funktion. Diese besteht zunächst einmal darin, dass dem Wunschgegenstand/Wertobjekt gewissermaßen positive oder negative Wertpunkte gegeben werden, die das Prima-facie-Interesse des Subjekts an diesem Gegenstand ausdrücken. Welchen Sinn haben diese Punkte? Menschen können durch ihr Handeln Wünsche gezielt realisieren. Aber sie haben sehr viele Wünsche und Interessen, die sie bei weitem nicht alle realisieren können, die meisten überhaupt nicht und von den verbleibenden Wünschen den größten Teil nur in Konkurrenz zur Realisierung anderer Wünsche. Ab einem gewissen Alter wissen Menschen dies. Sie reagieren darauf, indem sie sich zum einen um eine Verbesserung ihrer Handlungsmöglichkeiten bemühen und zum anderen in Deliberationen mittels Bewertungen von Handlungen und ihrer Folgen anhand eben jener Wertpunkte zu bestimmen versuchen, welche Handlungen denn, wenn schon nicht alle, dann wenigstens am meisten oder die wichtigsten der eigenen Wünsche realisieren würden. Diejenige Handlung, die dabei am höchsten bewertet wird, wird ausgeführt (Davidson 1980b, S. 98-100; Lumer 2005a, S. 245-247; Überblicke über entsprechende psychologische und ökonomische Entscheidungsmodelle: Camerer 1995, S. 617-674; Crozier und Ranyard 1997). Diese (vereinfachte) Erklärung schreibt den Wünschen also nicht unmittelbar eine motivationale Funktion zu, sondern nur mittelbar als Beitrag zu dem am Ende der Deliberation gefällten Urteil, welche Handlung denn am besten ist. Das Fällen dieses Optimalitätsurteils stellt die Entscheidung dar; und das Optimalitätsurteil selbst ist die Absicht, die dann im Standardfall in eine Handlung umgesetzt wird. Die Absicht ist der eigentlich motivationale Zustand.

Wenn Wünsche und die aus ihnen entwickelten (motivationalen) Präferenzen und Optimalitätsurteile glaubensgleich oder gar Meinungen selbst sind, dann müssten ihre Inhalte, propositionalen Gehalte auch wahrheitsfähig sein, also kognitiv. In einem gewissen Sinn ist dies auch so: Ihr Inhalt ist, dass die Wertobjekte die praktischen, motivationalen Standards des Wertsubjekts im angegebenen Maße erfüllen; deshalb können diese einen Wunsch ausdrückenden Werturteile durchaus falsch sein: Die vermeintlich gute Handlung erfüllt die motivationalen Standards des Wertsubjekts tatsächlich nicht in einem hohen Maß.

Nach der bei der Bildung und Begründung der Wünsche (und Präferenzen) berücksichtigten Wirkungsbreite der Wunschgegenstände muss formal zwischen 1. intrinsischen/letzten, 2. extrinsischen/instrumentellen, 3. totalen/Gesamt- und 4. prospektiven/Aussichtswünschen (und -präferenzen) unterschieden werden. 1. Ausgangspunkt sind intrinsische/letzte Wünsche, mit denen Dinge um ihrer selbst willen bewertet werden, unabhängig von ihren Folgen oder sonstigen Implikationen. Das intrinsisch Gewünschte (oder Abgelehnte) ist das, worum es dem Subjekt eigentlich geht. Die anderen drei Arten von Wünschen haben demgegenüber schon einen funktionalen Sinn: Sie sind Teil unserer rationalen Strategie, durch unsere Handlungen möglichst viel und möglichst wichtiges intrinsisch Gewünschtes zu realisieren und intrinsisch Abgelehntes zu verhindern. 2. Extrinsisch/instrumentell wünscht man einen Gegenstand $p$ im Maße $u$, wenn man annimmt, $p$ impliziere (z. B. verursache oder bedeute nach sozialen Regeln) einen im Maße $u$ intrinsisch ge- 
wünschten Gegenstand $q . p$ wird extrinsisch gewünscht, weil mit seiner Realisierung automatisch auch das intrinsisch Gewünschte $q$ realisiert wird. Um seinen eigentlichen Wunsch nach $q$ zu realisieren, braucht sich das Subjekt also nur um die Realisierung von $p$ zu kümmern. 3. Ein und derselbe Gegenstand $p$ kann mehrere intrinsisch gewünschte Gegenstände $q_{1}, q_{2}$ etc. implizieren. Das Subjekt $s$ wünscht dann $p$ insgesamt im Maße $u$, wenn $s$ annimmt, $u$ umfasse (idealiter: sei die Summe aller) die intrinsische Wünschbarkeit von $p$ selbst und die intrinsischen Wünschbarkeiten aller Implikationen von $p$. Der insgesamt gewünschte Gegenstand $p$ kann insbesondere eine eigene Handlung sein. Der $u$ starke totale Wunsch nach $p$ vereinfacht wieder die auf eine maximale Realisierung der eigenen Wünsche zielende Entscheidung: Die totale Wünschbarkeit gibt an, wieviel an intrinsischen Wünschbarkeiten, also von dem, was das Subjekt eigentlich interessiert, durch die Realisierung von $p$ mitrealisiert werden. Im Sinne der Optimierungsstrategie wird man dann diejenige Handlung ausführen, deren angenommene totale Wünschbarkeit am höchsten ist. 4. Meistens sind nicht alle relevanten Implikationen eines Wertgegenstandes sicher bekannt; das Subjekt nimmt nur an, dass der Wertgegenstand $p$ mit einer gewissen Wahrscheinlichkeit $w_{i}$ den Sachverhalt $q_{i}$ impliziert. Die Subjekte gehen mit dieser Situation meist so um, dass sie prospektive/Aussichtswünsche bilden: Diese rechnen dem Wertgegenstand $p$ nur einen Teil der intrinsischen Wünschbarkeit von $q_{i}$ zu, je geringer die Wahrscheinlichkeit ist, desto weniger. Die in instrumentellen, totalen und prospektiven Wünschen angenommenen Werturteile sind also durch Rekurs auf die in den intrinsischen Wünschen angenommenen Werturteile definiert; entsprechend sind die totalen und prospektiven Wünsche über intrinsische Wünsche und zusätzliche empirische Annahmen begründbar und sollten rationaliter auch entsprechend begründet sein. Und selbst die intrinsischen Wünsche haben ja den kognitiven Gehalt, dass der Wertgegenstand die Bewertungskriterien des Subjekts im angegebenen Maße erfüllt.

Die Kriterien für unsere intrinsischen (wirklich motivationalen) Wünsche ebenso wie das Streben nach Erfüllung möglichst vieler und wichtiger Wünsche sind weitgehend durch unsere psychische Struktur festgelegt und nicht kognitiv beeinflussbar. (Zu einigen doch vorhandenen Spielräumen s.: Lumer 2009, S. 169-182, 205-218.) In diesem Sinne sind unsere Wünsche nonkognitiv. Man muss also unterscheiden zwischen einem semantischen Kognitivismus der in unseren Wünschen enthaltenen Werturteile - diese Werturteile sind aufgrund unserer Kriterien für motivationale Wünschbarkeit wahrheitsfähig - und dem adoptiven Nonkognitivismus unserer Wünschbarkeitskriterien: Die Kriterien dafür, was wir praktisch (in der oben skizzierten Weise) entscheidungsbeeinflussend als so und so wünschbar annehmen, gehören zu unserer „motivationalen Mechanik“, die wir kognitiv nur wenig beeinflussen können. 


\section{$4 \quad$ Nutzen und Nutzenmessung}

,Nutzen' oder ,Wünschbarkeit‘ (,die Wünschbarkeit/der Nutzen des Gegenstandes/ Sachverhalts $p$ für das Subjekt $s^{`}$; formal: $U_{s} p$; dies drückt dann eine Zahl aus, z. B.: $U_{s} p=6$ ) ist ein quantitativer Begriff, der messen soll, was wirklich gut (oder schlecht) für ein Individuum ist - insbesondere auch in Abgrenzung von dem, was dieses Individuum selbst für gut hält oder wünscht. Dieser Begriff ist also einerseits in dem Sinne nicht subjektiv, sondern objektiv im Sinne von ,kognitiv ${ }^{6}$, dass es Kriterien für seine Zuschreibung gibt; andererseits ist er in einem doppelten Sinne doch subjektiv: Zum einen ist er subjektrelativ, bezieht sich auf ein Wertsubjekt , gut für $s^{6}$, zum anderen verwendet er als Grundlage für die Bewertung des für $s$ Guten doch, je nach Theorie in etwas unterschiedlicher Weise, die Einstellungen des Wertsubjekts $s$ selbst (Subjektautonomie). Die Gründe für dieses Wechselspiel sind: Die Subjektrelativität gibt wieder, dass verschiedene Subjekte unterschiedliche Interessen haben; was für den einen gut ist, kann für andere schlecht sein. Die Subjektautonomie beruht zum einen auf der liberalen Anerkennung der Autonomie des Subjekts: $s$ soll im Grunde selbst festlegen, was seine Interessen sind; alles andere wäre eine Bevormundung, Entfremdung, eventuell sogar Instrumentalisierung oder Ausbeutung (Williams 1981), gegebenenfalls auch metaphysisch leer. Zum anderen soll die Subjektautonomie die motivationale Wirksamkeit des Nutzenbegriffs garantieren: Nur wenn die Subjekte ihre Interessen in dem Nutzenbegriff aufgehoben sehen, werden sie ihn zur Grundlage ihrer Entscheidungen machen; denn Subjekte können sich in ihren Entscheidungen nur auf ihre motivationalen Präferenzen stützen, die aber bloß in engen Grenzen veränderbar sind (s.o., Abschn. 3) (Lumer 2009, S. 264-266, 325-327, 335-336, 338-340). Der Kognitivismus schließlich soll die epistemische Rationalität des Nutzenbegriffs garantieren: Menschen begehen bei der Bildung ihrer Wünsche und Präferenzen viele, sehr unterschiedliche epistemische Fehler oder verfügen nicht über hinreichendes Wissen, was alles im Nutzenbegriff korrigiert wird. - Der andere große Unterschied des Nutzenbegriffs zum Wunschbegriff ist, dass ersterer wirklich quantitativ, kardinal ist, also auf Intervall- oder Verhältnisskalenniveau gemessen wird, nicht nur wegen der größeren Präzision, sondern auch um - insbesondere bei seiner Verwendung in der Ethik - Addition und Multiplikation zu ermöglichen.

\subsection{Erwartungsnutzentheorie}

Der klassische Ansatz, Quantitativität und praktische Rationalität zusammenzubringen, ist die vor allem in der Mikroökonomie verwendete Erwartungsnutzentheorie von von Neumann und Morgenstern (1947). Während eine subjektive Schätzung (,mein Wunsch nach $p$ ist fünf Einheiten stark') keine kardinale Messung darstellt, kann man die kardinale Wunschstärke aber mit Hilfe von Präferenzen messen: Man befragt die Subjekte methodisch nach bestimmten ihrer Präferenzen, nämlich Präferenzen, die sich auf klar messbare Gegenstände beziehen, deren Wünschbarkeit aber vermutlich proportional zu diesen Messgrößen ist, nämlich Lotterien mit variablen 
Wahrscheinlichkeiten oder Gefühle unterschiedlicher Dauer: je höher die Gewinnwahrscheinlichkeit bzw. je länger die Dauer eines angenehmen Gefühls, desto besser. Wenn man hinreichend viele Präferenzen und dann auch Indifferenzen ermittelt hat, kann man die relativen Nutzen verschiedener Objekte etwa wie folgt berechnen: Das Wertsubjekt $s$ ist beispielsweise indifferent zwischen einer Lotterie $\alpha$, bei der es mit der Wahrscheinlichkeit $w_{a}$ den Preis $a$ erhält und mit der Wahrscheinlichkeit $1-w_{a}$ nichts, und einer Lotterie $\beta$, bei der es mit der Wahrscheinlichkeit $w_{b}$ den Preis $b$ erhält und anderenfalls nichts. Dann sollten die Stärken der Wünsche nach $a$ und $b$ sowie die Nutzen von $a$ und $b$ rationalerweise im Verhältnis $w_{b} / w_{a}$ zueinander stehen (denn $\left.U_{s} a \cdot w_{a}=U_{s} b \cdot w_{b} \Leftrightarrow U_{s} a / U_{s} b=w_{b} / w_{a}\right)$. Eine derartige Messung setzt allerdings voraus, dass die Präferenzen des Wertsubjekts eine Reihe starker Rationalitätsaxiome oder Kohärenzbedingungen erfüllen, u. a. Vergleichbarkeit/Vollständigkeit (dieses Axiom hat mehrere Namen), Transitivität, Abgeschlossenheit (wenn $a$ und $b$ mögliche Wertgegenstände sind, dann auch alle Lotterien, bei denen man entweder $a$ oder $b$ mit komplementären Wahrscheinlichkeiten erhält), Unabhängigkeit (wenn $a$ vor $b$ präferiert wird, genau dann wird auch eine Lotterie $\alpha$, in der mit der Wahrscheinlichkeit $w a$ eintritt, anderenfalls ein Objekt $c$, präferiert vor einer Lotterie $\beta$, in der mit der Wahrscheinlichkeit $w b$ resultiert, anderenfalls wieder das Objekt $c$ ) etc. (vereinfachte Axiomatisierung nach: Dawes 1988, S. 152). Wenn diese Axiome erfüllt sind, kann man die Entscheidungen des Subjekts $s$ als Maximierung des Erwartungsnutzens darstellen. (Seien $a$ und $b$ etc. mögliche Resultate der Lotterie $\alpha$, dann ist der Erwartungsnutzen von $\alpha: U_{e r w} \alpha=u_{a} \cdot w_{a}+$ $\left.u_{b} \cdot w_{b}+\ldots\right)$

Aber diese Rationalitätsaxiome sind so stark, dass niemand sie umfassend erfüllt, sondern allenfalls in kleinen Bereichen. Die Hauptgründe für diese „Irrationalität“ sind: 1. Zum einen gewichten die meisten Menschen bloß probabilistische Resultate nicht proportional zu ihrer Wahrscheinlichkeit, sondern gewichten kleine Wahrscheinlichkeiten über und sehr hohe Wahrscheinlichkeiten unter (Tversky und Kahneman 1992). 2. Zum anderen sind die vielen zu vergleichenden Lotterien so kompliziert und enthalten so viele potenzielle quantitative Werte, dass die Subjekte sie weder quantitativ ausdrücken noch, wenn sie alle quantitativ repräsentiert wären, in der benötigten Weise im Kopf verrechnen können; die Subjekte greifen deshalb bei der Entscheidung auf vereinfachende Heuristiken zurück, deren - relativ kleine Fehler von Entscheidungspsychologen dann gezielt ausgenutzt werden können, um die Probanden z. B. zu intransitiven Präferenzen zu verlocken (Slovic und Lichtenstein 1983). Solche Entscheidungsheuristiken werden sogar selbst nach ihrer Nützlichkeit für die weitestgehende Wunscherfüllung bewertet und weiterentwickelt (Payne et al. 1993; Lumer 2005a, S. 248-254). Es gibt also nicht das eine Entscheidungskriterium, das von Wünschen zu Entscheidungen führt. (Forschungsüberblick: Koehler und Harvey 2004.) Wenn die Subjekte aber nicht die von-NeumannMorgenstern-Axiome erfüllen, dann kann die Erwartungsnutzentheorie auch nicht zur Bestimmung ihrer Nutzenfunktionen verwendet werden - außer vielleicht mit Idealisierungen in bestimmten begrenzten Gebieten. Auch pragmatisch macht diese Idealisierung keinen Sinn: Wenn eine rationale Entscheidungstheorie manchmal ratlosen und öfter irrationalen Menschen dabei helfen soll, rationale Entscheidungen 
zu treffen, dann kann bei der Bereitstellung der Grundlagen für eine rationale Entscheidung nicht die umfassende Rationalität vorausgesetzt werden, die sogar impliziert, dass die erst zu treffende Entscheidung von derselben Person schon rational getroffen worden ist. Hinzu kommt, dass die Erwartungsnutzentheorie nur auf eine Kohärentisierung der Behandlung der Nutzen und Wahrscheinlichkeiten zielt; eventuelle empirische Fehlannahmen oder aus anderen Gründen korrekturbedürftige Wünsche werden dabei nicht einmal in Angriff genommen. (Lumer 1998, S. 33-38.).

\subsection{Philosophische Wünschbarkeitstheorien}

Philosophische Theorien epistemisch rationaler Wünschbarkeiten setzen vor allem an dem zuletzt genannten Problem empirisch falscher Informationen und der Verzerrung selbst intrinsischer Wünsche an. Sie beharren dabei weiter auf Subjektautonomie, die in den nicht kognitiven Komponenten unserer Wünsche steckt, identifizieren aber viel mehr - epistemische - Fehlermöglichkeiten oder Unterentwicklungen als die Erwartungsnutzentheorie, die keineswegs zur Autonomie beitragen, sondern im Sinne der epistemischen Rationalität beseitigt werden sollten. Wegen dieser Verschärfung der epistemischen Anforderungen an Wünschbarkeiten kann man statt bloß von einer ,rationalen“ auch von einer ,prudentiellen Wünschbarkeitstheorie“ sprechen. Das Ziel prudentieller Entscheidungen ist dann wieder, die so verbesserte (Aussichts-)Wünschbarkeit zu maximieren.

Es gibt zwei Grundansätze zu einer philosophischen prudentiellen Wünschbarkeitstheorie. 1. Der Volle-Informations-Ansatz (Brandt 1979, Teil 1; Griffin 1986; Sumner 1996) definiert die ,prudentielle Wünschbarkeit‘ über solche Präferenzen, die bei voller Information über alle für die Entscheidung relevanten Sachverhalte und bei deren lebhafter Repräsentation gebildet werden. Einige Probleme dieses Ansatzes sind: (i) Wir sind nie in der geforderten Weise voll informiert über alle möglichen Folgen. (ii) Wir können uns auch nicht alles Relevante auf einmal lebhaft vorstellen. (iii) Deshalb wissen wir auch nie, wie wir dann entscheiden würden; der Ansatz liefert deshalb keine sicheren Aussagen über prudentielle Wünschbarkeiten. (iv) Der Volle-Informations-Ansatz ist holistisch; er gibt keine Handreiche, wie wir Präferenzen bilden sollen; er definiert also nur den Input einer black box und verlässt sich dann auf den korrekten Output, sagt aber nichts über das Procedere und hilft damit nicht praktisch bei der Entscheidung.

2. Ein weiterer Ansatz der rationalen Wünschbarkeitstheorie ist analytisch-synthetisch (Lumer 2009, Kap. 4-5; 1998): Ausgangspunkt ist eine minutiöse Erforschung der alternativen Wege und Mechanismen und der fixen Grundlagen davon, wie Menschen tatsächlich Handlungen bewerten und entscheiden. Sodann wird unter diesen Alternativen nach dem Kriterium selektiert, welcher dieser Bewertungswege in der Weise aufklärungsstabil ist, dass er bei Konfrontation mit beliebigen (wahren) Informationen nicht aufgegeben wird. Schließlich wird, Wünschbarkeit' so definiert, dass zur Bestimmung dieser Wünschbarkeit der aufklärungsstabile Bewertungsweg gegangen werden muss. Insbesondere werden nach diesem Ansatz die 
hinter den intrinsischen Wünschen stehenden Kriterien als das Definiens der rationalen intrinsischen Wünschbarkeiten angenommen (vgl. auch Wessels 2011, S. 60); und es wird dann genau spezifiziert, wie daraus totale und Aussichtswünschbarkeiten so zu berechnen sind, dass das Streben nach maximaler Wunscherfüllung befriedigt wird. Die empirischen Informationen über die Implikationen des Bewertungsgegenstandes sind hingegen objektiv, müssen wahr sein. Dieser Ansatz garantiert zum einen ein Maximum an Subjektautonomie, indem er sich an dem orientiert, was dem Subjekt eigentlich wichtig ist, zum anderen ein Maximum an epistemischer Rationalität.

\section{$5 \quad$ Intrinsischer Nutzen und intrinsische Wünschbarkeit}

Nach der gerade skizzierten prudentiellen Nutzentheorie ist für die Nutzenbestimmung die Festlegung des Kriteriums für den intrinsischen Nutzen und dafür wiederum die Ermittlung der hinter unseren intrinsischen Wünschen stehenden Kriterien zentral. Obwohl dies in der Philosophie ein altes Thema ist, ist der Begriff des intrinsischen Nutzens in der Erwartungsnutzentheorie unbekannt (diese unterscheidet nicht zwischen basalen und abgeleiteten Präferenzen (Lumer 1998, S. 33-37)), wie auch in der Psychologie der Begriff des intrinsischen Wunsches nicht verwendet wird (der dort verwendete Begriff ,intrinsische Motivation` bezeichnet etwas Anderes).

Psychologischer Hedonismus ist die Theorie, dass allein unsere eigenen angenehmen Gefühle intrinsisch gewünscht und unsere unangenehmen intrinsisch abgelehnt werden. Rationaler Hedonismus ist die Theorie, dass eine solche Bewertung rational ist, also unseren intrinsischen Nutzen wiedergibt. Bentham vertrat einen psychologischen und einen rationalen Hedonismus, und zwar einen quantitativen Hedonismus, nach dem, in heutiger Terminologie rekonstruiert, die intrinsische Wünschbarkeit proportional ist zum Integral der gerichteten (positiven oder negativen) Intensität unserer (un-)angenehmen Gefühle über der Zeit (Bentham 1982, $\S \S$ I.1, I.5, III.1, IV.2). Es gibt viele Kritiken am quantitativen psychologischen Hedonismus und damit auch rationalen Hedonismus (Diskussion: Lumer 2009, S. 449-452, 494-496, 529-548). Die allermeisten beschränken sich aber auf den Versuch, zu beweisen, dass Menschen auch noch andere Dinge intrinsisch wünschen oder ablehnen als Freuden bzw. Leiden (das Wohl anderer, Wissen, Tugend, Gerechtigkeit, die Bestrafung von Übeltätern ... (z. B. Lumer 1997b)) oder dass der quantitative Hedonismus nicht ganz korrekt sein kann (durch Drogen oder sonstige Manipulation herbeigeführte angenehme Gefühle werden diskontiert ...). Sie greifen aber den Kern des psychologischen und rationalen quantitativen Hedonismus nicht an, sondern fordern im Prinzip nur gelegentliche Ergänzungen und Korrekturen der quantitativ hedonistischen Bewertung; und diese Ergänzungen und Korrekturen sind dann in der Regel viel umstrittener als der Kern des Hedonismus selbst. Einer der bekanntesten Reformvorschläge des rationalen Hedonismus stammt beispielsweise von John Stuart Mill. Er hat die rationale hedonistische Bewertung selbst verändern wollen durch den Übergang vom quantitativen zum qualitativen Hedo- 
nismus: Bei der Bewertung von Gefühlen solle neben deren Dauer und Intensität auch die Qualität im Sinne von niederen und höheren Freuden berücksichtigt werden (Mill 1976, S. 15, 19-20). Aber diesem Vorschlag ist kaum jemand gefolgt. Oder Ross sah außer den Gefühlen auch Tugend, die Verteilung von Freude und Leid sowie Wissen als intrinsische Güter an (Ross 2002, S. 134, 138-139). Aber auch seinem Vorschlag sind nicht viele gefolgt, u. a. mit dem Argument, Wissen und Tugend seien klar instrumentell und die Verteilung von Freude und Leid vielleicht ein moralisches Gut, aber kein prudentielles.

In der angewandten Ethik, die ihre Einschätzungen auf echte, empirisch untermauerte prudentielle Nutzen stützt, werden deshalb (anders als in der spekulativeren normativen oder Metaethik) fast durchgängig hedonistische Nutzenbestimmungen verwendet. (In der Medizinethik, teilweise auch in anderen angewandten Ethiken, werden beispielsweise als Werteinheiten QALYs, Quality Adjusted Life Years, benutzt, die neben der Dauer bestimmter persönlicher Zustände, z. B. eines Krankheitszustands, deren durch Präferenzen von Informierten gemessene Qualität berücksichtigen.)

\section{Nutzenaggregation als Definition des ,Gemeinwohls' und des ,moralischen Werts'}

In der Ethik wird der individuelle Nutzenbegriff häufig verwendet, um den ,moralischen Wert'/die ,moralische Wünschbarkeit' oder das ,Gemeinwohl' zu definieren. Und zwar werden, um den moralischen Wert von $p$ zu bestimmen, alle individuellen Nutzen von $p$ für die verschiedenen Subjekte aggregiert, d. h. die Gesamtmenge all dieser Nutzen wird mittels einer mathematischen Aggregationsfunktion - wie z. B. der Addition - auf einen einzigen Zahlenwert abgebildet; Ethiken, die die ,moralische Wünschbarkeit" so definieren, heißen „Wohlfahrtsethiken“. Im einfachsten Fall, nämlich dem Utilitarismus, ist die moralische Wünschbarkeit dann gleich der Summe der individuellen Nutzen. Der Sinn der Konzeption des moralischen Werts ist es, einen überindividuellen, sozialen und unparteiischen Wertmaßstab zu haben, mit dem - wenn ihm alle oder zumindest sehr viele eine überindividuelle Schiedsrichterfunktion zuschreiben - über gemeinschaftliche Projekte oder Interessenkonflikte entschieden werden kann. Gründe speziell für eine aggregierende moralische Wünschbarkeitsfunktion sind: 1. Der normative Individualismus dieser Konzeption reduziert alle Werte auf individuelle Nutzen. 1.1. Er realisiert damit vollständige Subjektautonomie. 1.2. Er lässt mit seiner klaren Grundlegung der individuelle Nutzen keinen Raum für metaphysische Wertkonzeptionen (wie ,das Volk' oder die ,höhere Ehre Gottes' ): Wert hat nur das, was gut für Individuen ist - wobei wir sehr gut verstehen, was dies bedeutet. 1.3. Dieser normative Individualismus erfasst zudem alles, was wirklich Wert hat. Er geht damit z. B. über ökonomische Gemeinwohlmessungen, wie etwa durch das Bruttoinlandsprodukt oder die Kosten-GewinnAnalyse, hinaus, die nur monetäre Werte erfassen oder, bestenfalls, nichtmonetäre Werte durch monetäre repräsentieren, dabei aber in jedem Fall den Beitrag des monetären Werts zum Wohle verfehlen müssen. (Denn monetäre Werte und Nutzen 
sind nicht proportional; und der Nutzen des Geldes ist einkommensabhängig.) 2. Gemeinwohl als Nutzenaggregation ist demokratisch und formal egalitär: Der Nutzen eines jeden zählt gleich viel. 3. Aggregative Wünschbarkeitsfunktionen haben wegen der für alle Nutzenvariationen sensiblen und paretanischen Aggregation von Quantitäten zu einer einzigen Quantität - nicht die von Arrow festgestellten Probleme einer Aggregation komparativer Präferenzen: Die resultierenden moralischen Wünschbarkeiten sind z. B. vollständig und streng transitiv. 4. Für die meisten Aggregationsmodi gibt es zudem noch speziellere Begründungen (s.u.).

\subsection{Utilitarismus}

Die älteste, ursprünglich von Bentham (1982, §§ I.2; I.4; IV.5) entwickelte, und verbreitetste Form der moralischen Nutzenaggregation ist der Utilitarismus: Zur Bestimmung des moralischen Werts eines Wertobjekts $p$ werden die Nutzen von $p$ für alle von $p$ Betroffenen addiert; dies ist der Nutzensummenutilitarismus (formal: $\left.U_{\text {mor } \text { utilit } \Sigma} p:=\Sigma_{i} U_{i} p\right)$. Eine Variante davon ist der Durchschnittsnutzentilitarismus, bei dem die Nutzensumme noch durch die Zahl der Betroffenen dividiert wird (formal: $U_{\text {mor,utilit } \varnothing p}:=1 / n \cdot \Sigma_{i} U_{i} p$ ).

Gegen den Utilitarismus sind viele Einwände erhoben worden; ein Teil davon richtet sich gegen die spezielle Aggregationsform. Die wichtigsten monieren, dass der Utilitarismus die Verteilungsgerechtigkeit völlig vernachlässige: 1. Wenn man zwei Nutzenverteilungen $a$ und $b$ hat mit: $a=\langle 1 ; 10\rangle ; b=\langle 0 ; 11,1\rangle$, so präferiert der Utilitarismus $b$, weil $b$ die höhere Nutzensumme aufweist, während die meisten Menschen intuitiv $a$ präferieren, weil dem ersten Subjekt $s_{1}$ aus der allerschlimmsten Not geholfen wird, während der etwas größere mögliche Nutzenzuwachs von Subjekt $s_{2}$ von 10 auf 11,1 als nicht so wichtig eingestuft wird. Die Gerechtigkeitsintuition hinter dieser Kritik zielt auf eine stärkere Berücksichtigung der schlecht Gestellten. 2. Der Utilitarismus würde von den Alternativen $c=\langle 5 ; 5\rangle ; d=\langle 0 ; 11\rangle$ wegen der höheren Nutzensumme die Option $d$ wählen und damit gegen egalitaristische Einstellungen entscheiden, nach denen die Beseitigung der krassen Ungleichheit in $d$ durchaus den Verlust einer Nutzeneinheit kosten darf. 3. Der Behinderte $s_{1}$ erziele bei gleichem Einkommen wegen der von ihm benötigten besonderen Aufwendungen jeweils nur die Hälfte des Nutzens, den der Vergnügungsmeister $s_{2}$ damit erzielt. Beim reinen Verteilungsproblem (also bei konstantem Geldbetrag) würde $s_{2}$ dann mehr Einkommen als der Behinderte bekommen, weil er damit mehr Nutzen erzeugt. Der Behinderte wäre doppelt schlecht dran: Er bekommt weniger Nutzen aus demselben Einkommen und er bekommt weniger Einkommen (Sen 1980, S. 478) - obwohl viele Menschen es gerechter fänden, wenn der Behinderte mehr Einkommen bekäme als der Gesunde, um seinen besonderen Bedarf decken zu können.

Alle anderen wohlfahrtsethischen Nutzenaggregationen versuchen, Komponenten der Verteilungsgerechtigkeit in die Aggregationsformel einzubauen. Die wichtigsten unter ihnen sind der (moderate Nutzen-)Egalitarismus, Leximin, der Prioritarismus und der Suffizientarismus. 


\subsection{Moderater Nutzenegalitarismus}

Nach dem moderaten Nutzenegalitarismus ist der moralische Wert einer Nutzenverteilung identisch mit ihrem utilitaristischen Wert (oft in der Durchschnittsnutzenvariante), von dem aber ein Ungleichheitsmaß subtrahiert wird. Solch ein Ungleichheitsmaß ist etwa die Standardabweichung $\sigma$. (Formal: $\sigma\left\langle u_{1} ; \ldots ; u_{n}\right\rangle:=\sqrt{ }\left(1 / n \cdot \Sigma_{i}\left(u_{\mu}-u_{i}\right)^{2}\right) ; u_{\mu}$ ist der Durchschnittsnutzen.) Rescher hat als egalitaristische moralische Nutzenaggregation das „effective-average-principle“ vorgeschlagen; dies ist der Durchschnittsnutzen minus der halben Standardabweichung (Rescher 1966, S. 33, 35). (Formal: $U_{\text {eap }}\left\langle u_{1} ; \ldots ; u_{n}\right\rangle=u_{\mu}$ $-1 / 2 \cdot \sigma\left\langle u_{1} ; \ldots ; u_{n}\right\rangle$.) Die Nutzenverteilung $\langle 2 ; 2 ; 2\rangle$ hat danach z. B. den egalitaristischen moralischen Wert 2 , die Nutzenverteilung $\langle 1 ; 2 ; 3\rangle$ den egalitaristischen Wert 1,59 und die Nutzenverteilung $\langle 0 ; 2 ; 4\rangle$ den egalitaristischen Wert 1,18-obwohl sie alle die gleiche Nutzensumme haben. Andere von Egalitaristen verwendete Ungleichheitsmaße sind z. B. der Gini-Koeffizient oder Gini-Koeffizient multipliziert mit dem Durchschnittsnutzen (Trapp 1988, S. 356). Temkin fordert die Berücksichtigung gleich einer ganzen Reihe von Ungleichheitsmaßen (Temkin 1993, Kap. 2). Kein namhafter Egalitarist schlägt die größtmögliche Nutzenegalität als alleiniges ethisches Bewertungskriterium vor; als zusätzliches Kriterium verwenden sie die Nutzensumme oder den Durchschnittsnutzen und vielleicht sogar noch weitere Kriterien. Dann müssen diese Teilkriterien im Gesamtkriterium gewichtet werden. Man sieht diese Gewichtung deutlich in Reschers Kriterium, in dem die zu subtrahierende Standardabweichung mit 1/2 multipliziert wird; sie könnte ja auch mit anderen Werten multipliziert werden. Eine Form des Nutzenegalitarismus heißt „moderat“, wenn diese Gewichte so gewählt sind, dass das Kriterium immer das Paretoprinzip erfüllt; $d$. h. eine Nutzenerhöhung für mindestens einen, bei der gleichzeitig niemand schlechter gestellt wird, bedeutet immer eine moralische Verbesserung - selbst wenn diese Nutzenerhöhung die Ungleichheit verstärkt.

Bekannte und prima facie plausible Kritiken am moderaten Nutzenegalitarismus sind: 1. Die Wahl des spezifischen Ungleichheitsmaßes und dessen Gewichtung müssen willkürlich bleiben. 2. Güter- oder Rechteegalitarismus mögen ja instrumentell häufig einen gewissen Sinn ergeben, weil überdurchschnittlicher Güter- oder Rechtebesitz für die einen bei reinen Verteilungsproblemen entsprechende Verluste für andere bedeuten. Dies gilt aber nicht für Nutzenungleichheit, weil man Nutzen nicht einfach von einer Person auf eine andere umverteilen kann. Was ist schlecht daran, wenn jemand wegen einer besonderen Glücksbegabung sich überdurchschnittlich wohlfühlt? 3. Der Egalitarismus ist ein vergleichendes Moralkriterium. Ist solch ein permanenter Vergleich nicht eher ein moralisch problematischer Charakterzug, der auf Neid beruht? Warum freut man sich nicht mit anderen, wenn es diesen gutgeht - solange dieses besondere Wohlsein nicht wieder auf übermäßigem Ressourcenkonsum beruht? 


\subsection{Maximin und Leximin}

Maximin ist ein Kriterium, nach dem der Wert einer Verteilung identisch ist mit dem Wert des schlechtesten Elements dieser Verteilung; der Maximin-Wert der Verteilung $a=\langle 1 ; 3 ; 6\rangle$ ist z. B. 1. Maximin bewertet alle Verteilungen mit dem gleichen niedrigsten Element gleich, auch wenn ansonsten große Unterschiede zwischen den Verteilungen bestehen; z. B. ist der Maximin-Wert von $b=\langle 1 ; 5 ; 8\rangle$ ebenfalls 1 . Leximin ist ein Kriterium, das diese Kontraintuitivität beseitigen soll. Leximin beginnt die Bewertung zunächst genau wie Maximin; wenn sich dann aber eine Parität ergibt, ist nach Leximin diejenige Verteilung besser, bei der das zweitniedrigste Element größer ist; bei $a$ und $b$ ist dies $b$, weil $5>3$; besteht auch dann noch Parität, richtet sich der Vorrang nach dem drittniedrigsten Element usw.

Rawls hatte Maximin als Kriterium für die Verteilung materieller Ressourcen vorgeschlagen (Rawls 1975, S. 336). Viele haben die Grundidee hinter diesem Vorschlag, dass es moralisch zuerst darauf ankommt, den am schlechtesten Gestellten zu helfen, plausibel gefunden, aber meist auch als zu radikal. Das Maximin- oder Leximinkriterium kann auch auf Nutzen, als der eigentlich grundlegenden Einheit, angewendet werden. Und so wird beim Vergleich von Gerechtigkeitskriterien auch Nutzenleximin diskutiert.

Allerdings ist Nutzenleximin de facto nur eine theoretische Möglichkeit; kein namhafter Ethiker vertritt dieses Prinzip (und noch weniger Nutzenmaximin). Denn Nutzenleximin interessiert sich praktisch (und Nutzenmaximin auch theoretisch) nur für die am schlechtesten Gestellten, ignoriert alle, auch riesige, Verbesserungen für etwas besser Gestellte und beutet diese gewissermaßen aus eben für die Verbesserung des Loses der am schlechtesten Gestellten.

\subsection{Prioritarismus}

Der Prioritarismus ist von diversen Theoretikern entwickelt worden (u. a. Atkinson und Stiglitz 1980; Boadway und Bruce 1984; Lumer 1997a, 2005b, 2009; McKerlie 1989, 1994; Nagel 1978; Parfit 1997; ausführliche Darstellung von Varianten und Diskussionen: Adler 2012, S. 307-551), vielfach mit der Intuition, eine Synthese aus der Idee hinter Leximin, nämlich eines Vorrangs für Verbesserungen für die schlechter Gestellten, allerdings in moderaterer Form, und dem utilitaristischen Effizienzdenken zu finden. Die Idee des Prioritarismus ist dann: Verbesserungen für schlechter Gestellte haben einen gewissen, aber keinen absoluten Vorrang vor Verbesserungen für Bessergestellte; je schlechter jemand gestellt ist, desto größer ist dieser Vorrang. Anders gesagt: Nutzenveränderungen für Menschen mit unterschiedlichen Nutzenniveaus haben unterschiedliches Gewicht; je schlechter jemand gestellt ist, desto größer ist dieses Gewicht. Parfits Slogan dafür ist: „Benefiting people matters more the worse off these people are" (Parfit 1997, S. 213). Verbesserungen für Gutgestellte können nach diesem Kriterium durchaus Verbesserungen für Schlechtgestellte moralisch vorgezogen werden, wenn sie viel größer sind oder viel mehr Personen zugutekommen. Technisch wird die moralische Wünschbarkeit 
nach dem Prioritarismus so bestimmt, dass auf die individuellen Nutzen eine moralische Bewertung angewendet wird, die zu dieser stärkeren Gewichtung von Verbesserungen für schlechter Gestellte führt; anschließend werden diese moralischen Bewertungen der individuellen Nutzen addiert, was dann die prioritaristische Wünschbarkeit ergibt. Diese Bewertungsfunktionen sind konkav wie in Abb. 1 dargestellt und führen deshalb zu der gewünschten Bewertungsart. Nach der Kurve $V P_{e 19}$ führt z. B. eine Nutzensteigerung (auf der $x$-Achse dargestellt) von 0 auf 0,1 zu einer Steigerung der moralischen Wünschbarkeit (auf der $y$-Achse dargestellt) von 0 auf 0,27 (+0,27), während eine Nutzensteigerung von 0,9 auf 1 zu einer moralischen Wünschbarkeitssteigerung von 0,98 auf 1 $(+0,02)$ führt, also zu einer 14 mal geringeren moralischen Wünschbarkeitssteigerung. Parfits Slogan, die Rede von den „Gewichten“, die die Nutzenveränderungen moralisch gesehen haben, bezieht sich hingegen auf die Steigung der moralischen Wünschbarkeitsfunktion, mathematisch gesprochen, also auf die erste Ableitung der Bewertungsfunktion, wie sie in Abb. 2 dargestellt ist: Dieses Gewicht sinkt also kontinuierlich bei immer höheren Nutzenwerten. Der Prioritarismus kann unterschiedlich stark sein. Dies ist aus Abb. 1 ersichtlich, in der mehrere prioritaristische Gewichtungsfunktionen aus einem Kontinuum eingezeichnet sind. (Die dort dargestellte Funktion ist exponentiell: $V P_{e e}(u)=(e /(e-1)) \cdot\left(1-e^{-u}\right)$, für $e>1 ; V P_{e 1}(u)=u$. Je größer der Koeffizient $e($ mit $e \geq 1)$ ist, desto radikalere Formen des Prioritarismus werden erzeugt.) Die Endpunkte des Spektrums sind die Diagonale $\left(V P_{e 1}\right)$ und eine Kurve, die sich durch immer weitere Ausbeulung nach oben links dem rechten Winkel durch die Punkte $\langle 0 ; 0\rangle,\langle 0 ; 1\rangle,\langle 1 ; 1\rangle$ beliebig annähert. Diese beiden Extreme sind der Utilitarismus (Diagonale) - der moralische Wert des Nutzens $u$ ist mit diesem Nutzenwert identisch, es gibt keine moralische Gewichtung - und Leximin - Annäherung an den rechten Winkel. Priorita-

Abb. 1 Exponentielle prioritaristische Bewertungsfunktionen: $V P_{e 1}$, $V P_{e 7}, V P_{e 19}, V P_{e 500}$ (Lumer 2005b, S. 28)

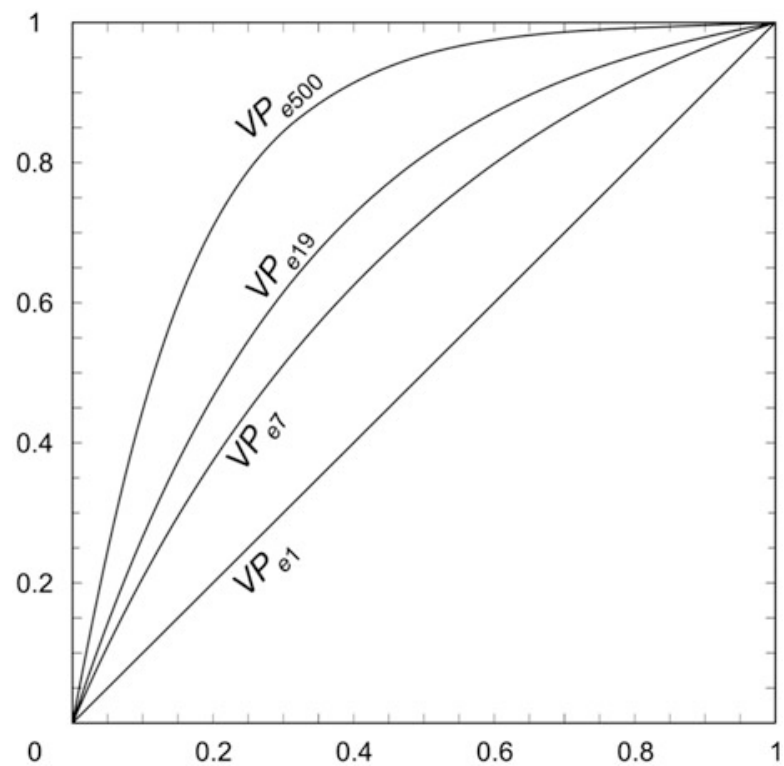


Abb. 2 Erste Ableitungen $V P_{e e}$ ' exponentieller prioritaristischer Bewertungsfunktionen (Lumer 2005b, S. 28)

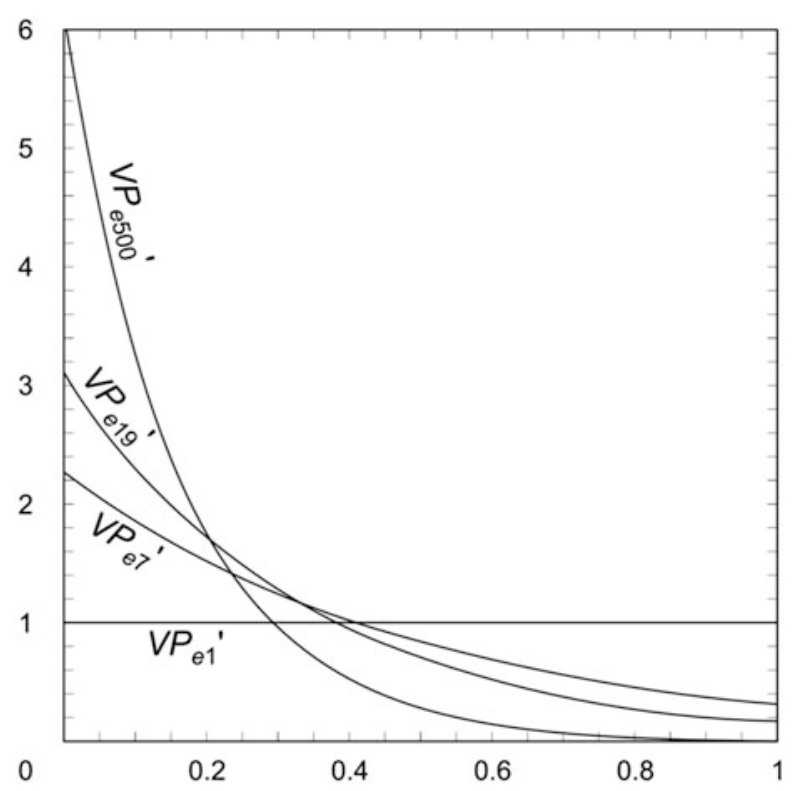

rismus ist nur das, was zwischen diesen Extremen liegt. Diese Verortung des Prioritarismus zeigt aber auch, dass er eine Synthese aus diesen beiden Kriterien darstellt: Er bewahrt die Leximin-Idee der Verteilungsgerechtigkeit durch stärkere Berücksichtigung der schlechter Gestellten, ohne aber die besser Gestellten und moralökonomische Effizienz zu vernachlässigen; und er übernimmt vom Utilitarismus die Effizienzidee sowie die Berücksichtigung aller, ist aber auch verteilungsgerecht. Für prioritaristische Bewertungen, die über Prinzipienaussagen hinausgehen und tatsächlich verschiedene Nutzenverteilungen quantitativ gegeneinander abwägen, muss die prioritaristische Bewertungsfunktion quantitativ bestimmt werden, also der Grad des Prioritarismus (der Koeffizent $e$ in der Bewertungsfunktion $V P_{e e}$ ) festgelegt werden (ebenso wie die Kurvenform). Über intuitive Festlegungen hinaus gibt es bislang erst eine Begründung einer quantitativen prioritaristischen Bewertungunsfunktion: eine Begründung des Prioritarismus mit der Bewertungsfunktion $V P_{e 19}$ über das Mitgefühl, das mit dieser Funktion optimiert werden soll (Lumer 2009, S. 589-632; Anwendung mit weiteren Kalibrierungen: Lumer 2002, insbesondere S. 26-32, 68-70). Die resultierende Bewertungsfunktion ist nicht linear, weil Mitleid intensiver ist als Mitfreude.

\section{$7 \quad$ Fazit}

Ziel dieses Artikels waren die Definitionen und Diskussion wohlfahrtsethischer Kriterien für das Gemeinwohl. Weil dies nutzenaggregierende Kriterien sind, wurden zunächst die vorausgesetzten Begriffe ,Präferenz‘, ,Wunsch“, ,(individueller) 
Nutzen' diskutiert und definiert. Die philosophischen Nutzentheorien sind quantitative Theorien des (individuellen) prudentiell Guten und versuchen, Subjektautonomie mit maximaler epistemischer Rationalität zu verbinden; der analytisch-synthetische Ansatz löst dabei einige Probleme des Volle-Informations-Ansatzes. Die materiale Anwendung dieser Theorien ergab dann, dass zumindest der Kern des intrinsischen Nutzens hedonistisch ist. Von den hier diskutierten wohlfahrtsethischen Kriterien der moralischen Wünschbarkeit oder des moralischen Werts hatten nur der Utilitarismus, der moderate Nutzenegalitarismus und der Prioritarismus eine hinreichend hohe Ausgangsplausibilität. Davon wird der Utilitarismus u. a. wegen des Fehlens der Verteilungsgerechtigkeit kritisiert, der Egalitarismus wegen seiner unzureichenden Begründung.

Abschn. 6 hat also bis zur Definition moralischer Wünschbarkeitskriterien geführt. Der nächste Schritt sind Handlungsempfehlungen und moralische Gebote. Dieser Schritt beinhaltet keineswegs automatisch, dass aus moralischer Sicht immer die moralisch beste Alternative realisiert werden muss - auch wenn manche Ethiken dies insinuieren. Eine solche Optimierungspflicht ist vielmehr handlungsutilitaristisch oder allgemeiner: handlungswelfaristisch. Alternativen dazu können z. B. die Pflicht zur Befolgung sozial geltender und moralisch guter Normen vorsehen oder die vorgeordnete Garantie von gewissen Rechten, z. B. um den Gesellschaftsmitgliedern einen dauernden Grund für ihre Kooperation zu geben.

Eine große Herausforderung für die normative Ethik bleibt die Pluralität der (nutzenaggregierenden) moralischen Wünschbarkeitskriterien - die in Abschn. 6 präsentierten sind nur die wichtigsten aus einer viel größeren Menge. Oft führen diese zwar zu ähnlichen Präferenzordnungen, oft aber auch nicht. Eine Antwort darauf ist, die zuletzt ziemlich vernachlässigte Begründung solcher Kriterien zu intensivieren, um auf diese Weise Entscheidungen zwischen ihnen zu fällen. Mindestens mittelfristig wird aber empirisch eine Pluralität von Ethiken vertreten werden; letztlich bleibt dann nur eine demokratische Wahl zwischen deren Empfehlungen. Die individuellen Entscheidungen bei solchen Wahlen werden sich dann empirisch aus einer Mischung prudentieller und persönlicher moralischer Überlegungen ergeben mit intersubjektiv unterschiedlicher Gewichtung dieser beiden Komponenten.

\section{Literatur}

Adler, Matthew D. 2012. Well-being and fair distribution. Beyond cost-benefit analysis. Oxford: Oxford University Press.

Atkinson, Anthony B., und Joseph E. Stiglitz. 1980. Lectures on public economics. London: McGraw-Hill.

Bentham, Jeremy. 1982 [1780/1789]. In An introduction to the principles of morals and legislation, Hrsg. J. H. Burns und H. L. A. Hart. London: Methuen.

Boadway, Robin W., und Neil Bruce. 1984. Welfare economics. Cambridge, MA: Basil Blackwell. Brandt, Richard B. 1979. A theory of the good and the right. Oxford: Clarendon.

Camerer, Colin F. 1995. Individual decision making. In The handbook of experimental economics, Hrsg. John H. Kagel und Alvin E. Roth, 587-703. Princeton: Princeton University Press. 
Crozier, Ray, und Rob Ranyard. 1997. Cognitive process models and explanations of decision making. In Decision making. Cognitive models and explanations, Hrsg. Rob Ranyard, Ray Crozier und Ola Svenson, 3-20. Oxford: Routledge.

Davidson, Donald. 1980a. Actions, reasons, and causes. In Essays on actions and events, 3-19. Oxford: Clarendon.

Davidson, Donald. 1980b. Intending. In Essays on actions and events, 83-102. Oxford: Clarendon.

Dawes, Robyn M. 1988. Rational choice in an uncertain world. Fort Worth: Harcourt Brace Jovanovich.

Fehige, Christoph, und Heinz Weiß. 2004. Wunsch. In Historisches Wörterbuch der Philosophie, Hrsg. Joachim Ritter, Karlfried Gründer und Gottfried Gabriel, Bd. 12, Sp, 1077-1088. Basel: Schwabe.

Griffin, James. 1986. Well-being. Its meaning, measurement, and moral importance. Oxford: Clarendon.

Grimm, Jacob, und Wilhelm Grimm. 1991 [1960]. Deutsches Wörterbuch. 14. Bd., II. Abteilung: Wilb-Ysop. Bearbeitet von Ludwig Sütterlin und den Arbeitsstellen des Deutschen Wörterbuches zu Berlin und Göttingen, Nachdruck Bd. 30. München: Deutscher Taschenbuch Verlag.

Koehler, Derek J., und Nigel Harvey, Hrsg. 2004. Blackwell handbook of judgment and decision making. Oxford: Blackwell.

Lumer, Christoph. 1997a. Utilex - Verteilungsgerechtigkeit auf Empathiebasis. In Current issues in political philosophy. Justice in society and world order, Hrsg. Peter Koller und Klaus Puhl, 99-110. Wien: Hölder-Pichler-Tempsky.

Lumer, Christoph. 1997b. The content of originally intrinsic desires and of intrinsic motivation. Acta Analytica 18:107-121.

Lumer, Christoph. 1998. Which preferences shall be the basis of rational decision? In Preferences, Hrsg. Christoph Fehige und Ulla Wessels, 33-56. Berlin/New York: de Gruyter.

Lumer, Christoph. 2002. The greenhouse. A welfare assessment and some Morals. Lanham/New York/ Oxford: University Press of America.

Lumer, Christoph. 2005a. Intentions are optimality beliefs - But optimizing what? Erkenntnis 62:235-262.

Lumer, Christoph. 2005b. Prioritarian welfare functions - An elaboration and justification. Working paper University of Siena, Department of Philosophy and Social Sciences. http://www.lumer. info/wp-content/uploads/2020/07/A066_Lumer_PrioritarianWelfareFunctions.pdf. Zugegriffen am 26.11.2020.

Lumer, Christoph. 2009. Rationaler Altruismus. Eine prudentielle Theorie der Rationalität und des Altruismus. 2., durchgesehene u. erg. Aufl. Paderborn: mentis.

McKerlie, Dennis. 1989. Equality and time. Ethics 99(3): 475-491.

McKerlie, Dennis. 1994. Equality and priority. Utilitas 6(1): 25-42.

Mele, Alfred R. 1992. Springs of action. Understanding intentional behavior. New York/Oxford: Oxford University Press.

Mill, John Stuart. 1976. Der Utilitarismus. Übersetzung, Anmerkungen und Nachwort von Dieter Birnbacher. Stuttgart: Reclam.

Nagel, Thomas. 1978. The justification of equality. Critica 10(28): 3-31.

Neumann, John von, und Oskar Morgenstern. 1947. Theory of games and economic behavior. Princeton: Princeton University Press.

Parfit, Derek. 1997. Equality and priority. Ratio 10:202-221.

Payne, John W., James R. Bettman, und Eric J. Johnson. 1993. The adaptive decision maker. Cambridge: Cambridge University Press.

Rawls, John. 1975. Eine Theorie der Gerechtigkeit. Frankfurt: Suhrkamp.

Rescher, Nicholas. 1966. Distributive justice. A constructive critique of the utilitarian theory of distribution. Indianapolis/New York/Kansas City: Bobbs-Merrill.

Ross, William David. 2002. The right and the good. Oxford: Clarendon.

Schroeder, Tim. 2020. Desire. In The Stanford encyclopedia of philosophy, Hrsg. Edward N. Zalta. https://plato.stanford.edu/archives/sum2020/entries/desire/. Zugegriffen am 26.11.2020. 
Schueler, George F. 1995. Desire. Its role in practical reason and the explanation of action. Cambridge, MA: MIT Press.

Sen, Amartya. 1980. Equality of what? In The Tanner lectures on human values, Hrsg. Sterling M. McMurrin, Bd. I, 197-220. Cambridge: Cambridge University Press.

Slovic, Paul, und Sarah Lichtenstein. 1983. Preference reversal. A broader perspective. American Economic Review 73(4): 596-605.

Sumner, L. W. 1996. Welfare, happiness, and ethics. Oxford: Clarendon.

Temkin, Larry S. 1993. Inequality. New York/Oxford: Oxford University Press.

Trapp, Rainer W. 1988. „,Nicht-klassischer “ Utilitarismus. Eine Theorie der Gerechtigkeit. Frankfurt: Klostermann.

Tversky, Amos, und Daniel Kahneman. 1992. Advances in prospect theory. Cumulative representation of uncertainty. Journal of Risk and Uncertainty 5:297-323.

Wessels, Ulla. 2011. Das Gute. Wohlfahrt, hedonisches Glück und die Erfüllung von Wünschen. Frankfurt: Klostermann.

Williams, Bernard. 1981. Internal and external reasons. In Moral luck. Philosophical papers 19731980, 101-113. Cambridge: Cambridge University Press. 